\title{
Astrocyte, reactive astrocytes and self-regulative apoptosis in the neuroinflammation
}

\author{
Liang-Wei Chen \\ Institute of Neurosciences, Department of Neurobiology and Collaborative Innovation Center for Brain Science, The Fourth \\ Military Medical University, Xi'an 710032, Shaanxi, China.
}

Astrocyte, one of the most abundant glial cell types, actively functions in stabilizing neural circuits and synaptic transmission in the central nervous system (CNS). Astrocytes not only provide metabolic and trophic supports to various CNS neurons and but also actively work in assisting synaptic transmission and plasticity. A line of growing evidences have documented that astrocytes present as an essential coordinatorin neural circuit function. ${ }^{[1]}$ Firstly, calcium signaling or calcium wave calcium $\left(\mathrm{Ca}^{2+}\right)$ between neighboring astrocytes contribute to establishment of a huge astrocytic glial network by gap-junctions, which has updated the understanding of astrocyte function in CNS, and led to an idea that astrocytes are powerful regulators of neuronal spiking, synaptic plasticity and brain blood flow as well. ${ }^{[2]} \mathrm{The}^{\mathrm{Ca}^{2+}}$ wave in astrocyte processes may also precede onset of hyperemia and function as regulators of neurovascular coupling. ${ }^{[3]}$ Secondly, astrocytes can also fast respond to sensory stimulation and involve in generation of neuronal rhythmic activity, and blockade with a $\mathrm{Ca}^{2+}$ chelator can sufficiently prevent neurons from a rhythmic bursting, indicating that astrocytes partially and critically contribute a fundamental neuronal firingpattern or generation of rhythmic activity. ${ }^{[4]}$ Thirdly, distinct astrocytic transporters like well-known glutamate transporter 1 (GLT-1) and dynamic diffusion play a physiological modulating role in shaping synaptic transmission between neurons. Glutamate action time

Corresponding Author: Dr. Liang-Wei Chen, Institute of Neurosciences, Department of Neurobiology and Collaborative Innovation Center for Brain Science, The Fourth Military Medical University, Xi'an 710032, Shaanxi, China. Email: Iwchen@fmmu.edu.cn

\begin{tabular}{|l|l|}
\hline \multicolumn{2}{|c|}{ Access this article online } \\
\hline Quick Response Code: & Website: \\
\hline & http://www.nnjournal.net \\
\hline
\end{tabular}

in synaptic transmission is controlled by the astrocytic GLT-1, i.e. while impairing GLT-1 diffusion could slow kinetics of excitatory currents or prolonged time course of synaptic glutamate transmission. ${ }^{\left[{ }^{[}\right]}$

Reactive astrocytes, a most common pathological hallmark, contribute to pathogenesis or progression of neurological disorders like trauma, ischemia, Alzheimer's and Parkinson's disease (PD). Astrocytes can in vitro and in vivo respond to various stimuli in trauma, ischemia and diseased conditions, fast change morphology and functional properties, and many appear asactivated or become reactive astrocytes. Reactive astrocytes undergo phenotypic changes and contribute to pathogenesis or progression of neurological disorders. For instance, those reactive astrocytes have been proposed to be incompetent bystanders in epileptogenesis as a result of cellular changes rendering them unable to perform housekeeping functions in diseased CNS. ${ }^{[6]}$ Astrocytes modulate excitatory and inhibitory balance by regulating astrocytic uptake of gamma amino acid butyric acid and glutamate efficiency. ${ }^{[7]}$ The reactive astrocytes or astrogliosis resulting from neuronal hyperexcitability further render inhibitory activity in epilepsy. New findings have thus challenged us to consider an important contribution of activated astroglial cells in epileptogenesis in the acquired epilepsy, although epilepsy has long been considered as a disease caused byabnormal increasing bursts of excitatory neurons, exclusively. ${ }^{[6,7]}$ In addition, the reactive astrocytes are characterized with high level of $G$ protein-coupled receptors such as adenosine receptor, which were

This is an open access article distributed under the terms of the Creative Commons Attribution-NonCommercial-ShareAlike 3.0 License, which allows others to remix, tweak, and build upon the work non-commercially, as long as the author is credited and the new creations are licensed under the identical terms.

For reprints contact: service@oaepublish.com

How to cite this article: Chen LW. Astrocyte, reactive astrocytes and self-regulative apoptosis in the neuroinflammation. Neuroimmunol Neuroinflammation 2016;3:167-9.

Received: 17-06-2016; Accepted: 22-06-2016 
evidently implicated in regulation of learning and memory activity in CNS. Conditional removal or down-regulation of these specific receptors enhanced memory in aging or Alzheimer's disease (AD) animals, indicating abnormal increase of adenosine receptor A2a in reactive astrocytes might contribute to AD-linked memory loss. ${ }^{[8]}$

Reactive astrocytes may display a selfregulativeapoptosis for modulation of overactivated astrocytes or functional balance in the neuroinflmmatory event or diseased CNS. Cell apoptosis was earlier identified in the reactive astrocytes, but its real significance remains unclear in the neuroinflammation and diseased conditions. Astrocytic apoptosis in vitro and in vivo might attribute to $\mathrm{Ca}^{2+}$ overload, mitochondrial dysfunction, oxidative stress and NF-kB signaling activation. ${ }^{\left[{ }^{[9]}\right.}$ It is known that obvious inflammatory injury of CNS neurons occur in pathogenesis of neurodegenerative diseases such as AD and PD. While the reactive astrocytes and microglial cells dominate in the inflammatory reaction, major histocompatibility complex II (MHC II) levels were significantly upregulated in midbrain of 1-methyl-4-phenyl-1,2,3,6tetrahydropyridine (MPTP) model of PD and MHC II was mainly localized in astrocytes and microglia, in which MHC II mediated T cell activation in initiating immune reaction and in participating disease progression. ${ }^{[10]}$ Besides, astrocyte activation and apoptosis was observed in lipopolysaccharide (LPS)triggered inflammation. ${ }^{[11]}$ Hyper- or over-activated astrocytes appear as hypertrophied morphology and glutamate transporter 1 up-regulation or functional changes that afterwards affect microglia-involving cytokine generation and inflammation. The reactive astrocytes underwent necroptosis in the animal model with spinal cord injury through M1 microglia/macrohpage-mediating way. ${ }^{[12]} \mathrm{By}$ in vivo and in vitro studies with LPS and MPTP models, we demonstrated that LPS plus cytokines or MPTP insult could result in obvious activation of astrocytes from the ventral midbrain and cerebral cortex, followed by appearance of apoptosis in a proportion of those reactive astrocytes. By mechanical analysis, apoptosis of reactive astrocytes was resulted from bax and cleaved caspase 3 up-regulation, and might be possibly related to significant up-regulation or activation of inducible gas-1 signaling. ${ }^{[13]}$ In addition, N-Myc Downstream-regulated gene 2 (NDRG2) was also found to involve in the astrocytic apoptosis and inhibition of NDRG2 expression reduced astrocytic apoptosis in ischemia. ${ }^{[14]}$ The ischemia-induced autophagy also influenced apoptosis of reactive astrocytes and down-regulation of autophagy caused time-dependent changes in extrinsic and intrinsic apoptotic pathways, indicating that autophagy in astrocytes might also act as an early adaptive response before initiation of apoptosis and necrosis. ${ }^{[15]}$

Taken together, we hypothesize that apoptosis of reactive astrocytes may also possibly present a self-regulator for those over-activated astrocytes or functional balance between neurotrophic and inflammatory properties in neuroinflammation, while reactive astrocytes work actively as aparticipatorinastrocyte-microglial communication and microglia-dominating inflammatory response. Nevertheless, one critical question regarding this self-regulatory apoptosis of reactive astrocytes in ameliorating the neuroinflammatory injury should still merit further extensive investigations to elucidate its exact roles in pathogenesis and disease progression of various neurological disorders.

\section{Financial support and sponsorship}

Supported by grants from the National Natural Science Foundation of China (31371374) and the National Basic Research Program (2012CB525002).

\section{Conflicts of interest}

There are no conflicts of interest.

\section{REFERENCES}

1. Khakh BS, Sofroniew MV. Diversity of astrocyte functions and phenotypes inneural circuits. Nat Neurosci 2015;18:942-52.

2. Bazargani N, Attwell D. Astrocyte calcium signaling: the third wave. Nat Neurosci 2016;19:182-9.

3. Otsu Y, Couchman K, Lyons DG, Collot M, Agarwal A, Mallet JM, Pfrieger FW, Bergles DE, Charpak S. Calcium dynamics in astrocyte processes duringneurovascular coupling. Nat Neurosci 2015; 18:210-8

4. Morquette P, Verdier D, Kadala A, Féthière J, Philippe AG, Robitaille R, Kolta A. An astrocyte-dependent mechanism for neuronal rhythmogenesis. Nat Neurosci 2015;18:844-54.

5. Murphy-Royal C, Dupuis JP, Varela JA, Panatier A, Pinson B, Baufreton J, Groc L, Oliet SH. Surface diffusion of astrocytic glutamate transporters shapessynaptic transmission. Nat Neurosci 2015;18:219-26.

6. Robel S, Sontheimer H. Glia as drivers of abnormal neuronal activity. Nat Neurosci 2016;19:28-33.

7. Muthukumar AK, Stork T, Freeman MR. Activity-dependent regulation of astrocyte GAT levels during synaptogenesis. Nat Neurosci 2014;17:1340-50.

8. Orr AG, Hsiao EC, Wang MM, Ho K, Kim DH, Wang X, Guo W, Kang J, Yu GQ, Adame A, Devidze N, Dubal DB, Masliah E, Conklin BR, Mucke L. Astrocytic adenosinereceptor A2A and Gscoupled signaling regulate memory. Nat Neurosci 2015;18:423-34.

9. Takuma K, Baba A, Matsuda T. Astrocyte apoptosis: implications forneuroprotection. Prog Neurobiol 2004;72:111-27.

10. Martin HL, Santoro M, Mustafa S, Riedel G, Forrester JV, Teismann $\mathrm{P}$. Evidence for a role of adaptive immune response in the disease 
pathogenesis of the MPTP mouse model of Parkinson's disease. Glia 2016;64:386-95.

11. Sharma A, Patro N, Patro IK. Lipopolysaccharide-induced apoptosis ofastrocytes: therapeutic intervention by Minocycline. Cell Mol Neurobiol 2016;36:577-92.

12. Fan H, Zhang K, Shan L, Kuang F, Chen K, Zhu K, Ma H, Ju G, Wang YZ. Reactive astrocytes undergo M1 microglia/macrohpagesinduced necroptosis in spinal cordinjury. Mol Neurodegener 2016;11:14.

13. Sun XL, Chen BY, Zhao HK, Cheng YY, Zheng MH, Duan L, Jiang W, Chen LW. Gas1 up-regulation is inducible and contributes to cell apoptosis in reactive astrocytes in the substantianigra of LPS and MPTP models. J Neuroinflammation 2016;13:180.

14. Ma YL, Zhang LX, Liu GL, Fan Y, Peng Y, Hou WG. N-Myc Downstream-regulatedgene 2 ( $\mathrm{Ndrg} 2)$ is involved in ischemiahypoxia-induced astrocyte apoptosis: a novel target for stroke therapy. Mol Neurobiol 2016; doi: 10.1007/s12035-016-9814-5.

15. Kasprowska D, Machnik G, Kost A, Gabryel B. Time-dependent changes in apoptosis upon autophagy inhibition in astrocytes exposed to oxygen and glucose deprivation. Cell Mol Neurobiol 2016; doi: 10.1007/s10571-016-0363-2 